\title{
Simulation Study of Real Time 3-D Synthetic Aperture Sequential Beamforming for Ultrasound Imaging
}

Hemmsen, Martin Christian; Rasmussen, Morten Fischer; Stuart, Matthias Bo; Jensen, Jørgen Arendt

Published in:

Proceedings of the SPIE - Progress in Biomedical Optics and Imaging

Link to article, DOI:

$10.1117 / 12.2044017$

Publication date:

2014

Document Version

Early version, also known as pre-print

Link back to DTU Orbit

Citation (APA):

Hemmsen, M. C., Rasmussen, M. F., Stuart, M. B., \& Jensen, J. A. (2014). Simulation Study of Real Time 3-D Synthetic Aperture Sequential Beamforming for Ultrasound Imaging. In Proceedings of the SPIE - Progress in Biomedical Optics and Imaging (Vol. 9040). [90401K] SPIE - International Society for Optical Engineering. https://doi.org/10.1117/12.2044017

\section{General rights}

Copyright and moral rights for the publications made accessible in the public portal are retained by the authors and/or other copyright owners and it is a condition of accessing publications that users recognise and abide by the legal requirements associated with these rights.

- Users may download and print one copy of any publication from the public portal for the purpose of private study or research.

- You may not further distribute the material or use it for any profit-making activity or commercial gain

- You may freely distribute the URL identifying the publication in the public portal 
Paper presented at the SPIE Medical Imaging 2014 Conference:

\section{Simulation Study of Real Time 3-D Syn- thetic Aperture Sequential Beamforming for Ultrasound Imaging}

Martin Christian Hemmsen, Morten Fischer Rasmussen, Matthias Bo Stuart and Jørgen Arendt Jensen

Center for Fast Ultrasound Imaging,

Biomedical Engineering Group,

Department of Electrical Engineering

Ørsteds Plads Building 349,

Technical University of Denmark, 2800 Kgs. Lyngby, Denmark. 


\title{
Simulation study of real time 3-D Synthetic Aperture Sequential Beamforming for Ultrasound imaging
}

\author{
Martin Christian Hemmsen, Morten Fischer Rasmussen, Mathias Bo Stuart, and \\ Jørgen Arendt Jensen \\ Center for Fast Ultrasound Imaging, Dept. of Electrical Engineering \\ Technical University of Denmark, 2800 Lyngby, Denmark.
}

\begin{abstract}
This paper presents a new beamforming method for real-time three-dimensional (3-D) ultrasound imaging using a 2-D matrix transducer. To obtain images with sufficient resolution and contrast, several thousand elements are needed. The proposed method reduces the required channel count from the transducer to the main imaging system, by including electronics in the transducer handle. The reduction of element channel count is achieved using a sequential beamforming scheme. The beamforming scheme is a combination of a fixed focus beamformer in the transducer and a second dynamic focus beamformer in the main system. The real-time imaging capability is achieved using a synthetic aperture beamforming technique, utilizing the transmit events to generate a set of virtual elements that in combination can generate an image. The two core capabilities in combination is named Synthetic Aperture Sequential Beamforming (SASB). Simulations are performed to evaluate the image quality of the presented method in comparison to Parallel beamforming utilizing 16 receive beamformers. As indicators for image quality the detail resolution and Cystic resolution are determined for a set of scatterers at a depth of $90 \mathrm{~mm}$ for elevation and azimuth angles from $0^{\circ}$ to $25^{\circ}$. Simulations show that the acoustic performance of the proposed method is less angle dependent than Parallel beamforming. The Cystic resolution is shown to be more than $50 \%$ improved, with a detail resolution on the same order as Parallel Beamforming.
\end{abstract}

Keywords: Real-time 3-D imaging, channel reduction, 2-D matrix transducer, Synthetic aperture, Sequential beamforming, Medical imaging

\section{INTRODUCTION}

In 3-D ultrasound imaging, an entire volume is imaged and allows the user to interactively control and manipulate the visualized planes to explore the entire volume while it is being updated in real time. The extension from 2-D to 3-D imaging greatly increases the number of lines to be acquired, leading to a proportional decrease in frame rate. Earlier suggestions to reduce the data acquisition time for a 1D-linear phased array is to increase the angular spread of the transmit beam and by simultaneously form adjacent receive beams. ${ }^{1,2}$ Von Ramm and Smith $^{3,4}$ developed the first real-time 3-D imaging system using a 2-D transducer array. The system applied a parallel beamforming technique that permitted the formation of many simultaneous receive beams throughout the volume interrogated by the transmit beam.

An alternative technique to solve these problems can be provided by synthetic aperture (SA) imaging. ${ }^{5-7} \mathrm{~A}$ key feature of SA imaging is that the transmissions and the size of the acquired volume are decoupled. In SA imaging a spherical wave is transmitted to collect information from a large region. The use of de-focused transmits has so far been the most common way of generating spherical waves. ${ }^{8-10}$ The final image is then created from the sum of the beamformed energy from multiple transmit bursts, where the origin of the transmitted wavefront is considered to be a virtual element. In essence, a synthetic aperture image is optimally focused at each pixel in the image, and the extent of the point spread function is determined by the density and spread of the virtual sources. One problem to be solved in SA imaging is how to reduce the number of transmission channels between the probe and the processing unit.

Further author information: Send correspondence to M. C. Hemmsen. E-mail: mah@elektro.dtu.dk 
A solution to this problem can be provided by synthetic aperture sequential beamforming (SASB). ${ }^{11} \mathrm{~A}$ key feature of SASB imaging is that only a single channel is required between the transducer and the main processing unit. Previous studies have shown that SASB imaging is superior to dynamic receive focusing for $2-\mathrm{D}$ imaging. ${ }^{12}$

This paper presents a new beamforming method for real-time three-dimensional (3-D) ultrasound imaging using a $64 \times 642$-D matrix transducer. To obtain images with sufficient resolution and contrast, several thousand elements are needed. The proposed method reduces the required channel count from the transducer to the main imaging system, to a single channel.The method is presented and computer simulations are used to evaluate the image quality in terms of Cystic- and detail resolution. The method is compared to 3-D parallel receive beamforming, and a simulation of an anechoic cyst is performed to link the image quality metrics to a visual comparison.

\section{SYNTHETIC APERTURE SEQUENTIAL BEAMFORMING}

This section describes the principle of Synthetic Aperture Sequential Beamforming (SASB). The method is a dual-stage procedure using two separate beamformers.

\subsection{First stage beamforming}

In the initial stage the beamformer operates on the signals received by the transducer array. The emission sequence scans a focused emission across the volume being imaged. The beamformer applies delay-and-sum beamforming with a fixed delay for each transducer element to create a fixed focus scan line. The delay configuration is identical in both transmit and receive. The sample values of a single scan line can be interpreted as the response from a virtual source, emitting a spherical wave, positioned at the focal point of the scan line. The response is offset in time, relative to the distance from the virtual source to the scan line reference point. In the following, $M$ emissions are used each having a unique direction $\theta_{m}$. For a given point $\vec{r}_{f p}$ on the scan line in direction $\theta_{m}$, the output of the beamformer is

$$
l_{\theta_{m}}\left(t_{d, \theta_{m}}\left(\vec{r}_{f p}\right)\right)=\sum_{k=1}^{K} \sum_{n=1}^{N} \mathcal{W}(k, n) R_{\theta_{m}, k, n}\left(t_{d, \theta_{m}}\left(\vec{r}_{f p}\right)\right)
$$

where $R_{\theta_{m}, k, n}$ is the measured response of the element with row index $k$ and column index $n$, and $\mathcal{W}$ is the weighting function of the receive aperture. The delay value, $t_{d}$, applied to the response from the element at physical position, $\vec{r}_{r}$, is determined by the time-of-flight calculation as

$$
t_{d, \theta_{m}}\left(\vec{r}_{f p}\right)=\frac{1}{c}\left(\left|\vec{r}_{t f p}-\vec{r}_{e}\right| \pm 2\left|\vec{r}_{f p}-\vec{r}_{t f p}\right|+\left|\vec{r}_{r}-\vec{r}_{t f p}\right|\right)
$$

where the \pm refers to whether the sample position is above or below the focus position, $\vec{r}_{t f p}$, and $\vec{r}_{e}$ is the scan line reference position for the unique direction $\theta_{m}$. Fig. 1 illustrates the time-of-flight calculation for the SASB first stage beamformer. Note from the figure that as the receive delay profile is fixed, the time delay offset is only relative to the distance from the virtual source to the receive focus point, $\vec{r}_{f p}$.

\subsection{Second stage beamforming}

The second stage beamformer takes the output of the first stage and uses it as input. The focal point from the first stage beamformer is considered a virtual source, while the samples on the scan line are considered the signal received by a virtual receive element colocated with the virtual source. Each point in the focused image line contains information from a set of spatial positions limited by the opening angle of the virtual source. The opening angle is defined as

$$
\alpha=2 \arctan \frac{1}{2 F \#}
$$

A single image point is therefore potentially represented in multiple first stage focused scan lines. The second stage beamformer creates a set of image points by combining information from the first stage focused scan lines 


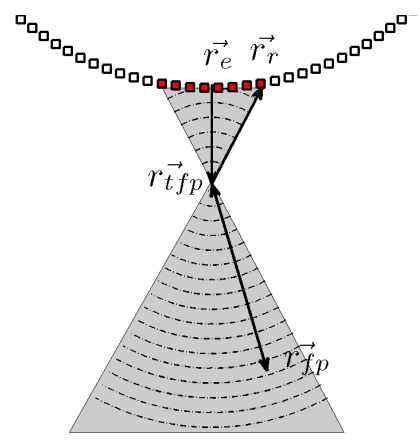

(a) SASB

Figure 1: Wave propagation path. The dashed lines indicate the propagating waves for a single multi element emission. The white squares indicate the individual transducer elements of a 1D transducer, and the red squares the active elements. The wave propagation path is shown as a solid line with arrow head, to indicate direction. The wave propagation path is used to determine the time-of-flight value that is applied in the first stage beamformer.

that contain information from the spatial position of the image point. A single sample, $h$, representing the image point at the location $\vec{r}_{i p}$ can be expressed as

$$
h\left(\vec{r}_{i p}\right)=\sum_{m=1}^{M} \mathcal{W}\left(\theta_{m}, \vec{r}_{i p}\right) l_{\theta_{m}}\left(t_{d, \theta_{m}}\left(\vec{r}_{i p}\right)\right),
$$

where $l_{\theta_{m}}\left(t_{d, \theta_{m}}\left(\vec{r}_{i p}\right)\right)$ is the sample value at time $t_{d, \theta_{m}}$ from the scan line with propagation direction $\theta_{m}$. The variable $\mathcal{W}$ is an apodization function, which controls the weighting of the virtual sources. The time delay, $t_{d, \theta_{m}}$ for the scan line with direction $\theta_{m}$ is found from the time-of-flight calculation as

$$
t_{d, \theta_{m}}\left(\vec{r}_{i p}\right)=\frac{2}{c}\left(\left|\vec{r}_{v s_{m}}-\vec{r}_{\theta_{m}}\right| \pm\left|\vec{r}_{i p}-\vec{r}_{v s_{m}}\right|\right)
$$

where $\vec{r}_{\theta_{m}}$ is the scan line reference position, and $\vec{r}_{v s_{m}}$ is the position of the virtual source.

The variable $\mathcal{W}$ in $(4)$ is an apodization function determining the weighting of the individual scan lines. The weight for the scan line, $l_{\theta_{m}}$ contributing to image point $\vec{r}_{i p}$ can be calculated from (6)

$$
\mathcal{W}\left(\theta_{m}, \vec{r}_{i p}\right)= \begin{cases}0.54+0.46 \cos (2 n \pi), & \text { if } n \text { is } \leq 0.5 \\ 0, & \text { if } n \text { is }>0\end{cases}
$$

for the case of a desired Hamming apodization, where $n$ can be calculated from

$$
n=\frac{d\left(l_{\theta_{m}}, \vec{r}_{i p}\right)}{\Delta}
$$

and is given as the ratio between the shortest distance, $d\left(l_{\theta_{m}}, \vec{r}_{i p}\right)$, from the image point, $\vec{r}_{i p}$, to the scan line, $l_{\theta_{m}}$, and a desired aperture width, $\Delta$. The distance $d\left(l_{\theta_{m}}, \vec{r}_{i p}\right)$ can be expressed as

$$
d\left(l_{\theta_{m}}, \vec{r}_{i p}\right)=\frac{\left\|\left(\vec{r}_{\theta_{m}}-\vec{r}_{V S_{m}}\right) \times\left(\vec{r}_{V S_{m}}-\vec{r}_{i p}\right)\right\|}{\left\|\vec{r}_{\theta_{m}}-\vec{r}_{V S_{m}}\right\|} .
$$

where $\times$ denotes the cross product. Fig. 2 b illustrates the apodization weight calculation.

\section{METHODS}

\subsection{Experiment setup}

To predict the beam pattern of the received field using SASB and Parallel Beamforming, simulations are performed with Field $\mathrm{II}^{13,14}$ and the Beamformation Toolbox III. ${ }^{15}$ In the simulations a $64 \times 64$ elements matrix transducer 


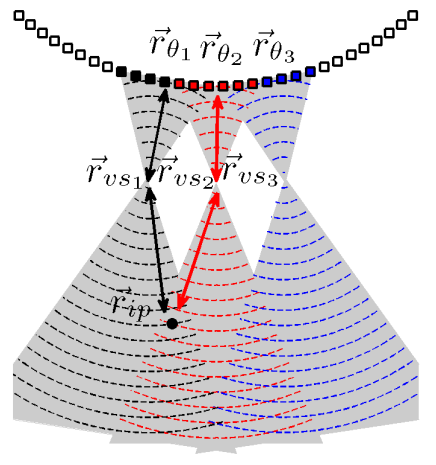

(a) Time-of-flight calculation

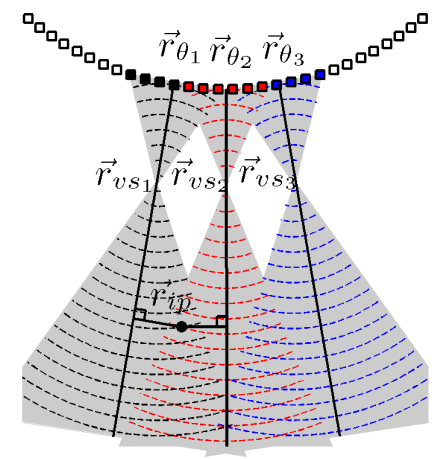

(b) Apodization weight calculation

Figure 2: In (a) and (b) the dashed lines indicate the propagating waves for three multi element emissions. The squares indicate the individual transducer elements of a 1D transducer. The black dot indicates a specific image point. In (a) the wave propagation path is shown as a solid line with arrow head, to indicate direction. In (b) the calculation of apodization weight is illustrated. In both (a) and (b) note that the third emission shown in blue does not contribute to the image point as it contains no information about the spatial position of the image point due to the limited opening angle.

with an element pitch of $300 \mu \mathrm{m}$ is considered. The elements are excited with a $3 \mathrm{MHz}$ two cycle sinusoidal pulse, shaped with a Hamming window. The element one-way impulse response is identical to the excitation pulse. Both imaging methods are set-up with a view field of $60 \times 60$ degrees and a scan depth of $10 \mathrm{~cm}$, resulting in 361 emissions for a frame rate of $20 \mathrm{~Hz}$. The 361 emissions are divided into 19 angles per dimension (elevation and azimuth), with uniform angle separation. Both imaging methods are implemented such that all scan lines have their reference point at the center of the transducer $(0,0,0)$. To have a realistic size of the cable connecting the ultrasound probe with the ultrasound scanner, the number of channels is limited to 256 . This does not influence the implementation af SASB, where only one channel exists between the ultrasound probe and the main processing unit, but is a restriction for parallel beamforming. For parallel beamforming, a limit of 256 cable channels means a limit of 256 active elements. See Fig. 3a for an illustration of the scan setup, including the physical and SASB-synthesized aperture.

\subsection{Implementation details}

The first stage beamformer is implemented using Field II and a sampling frequency of $120 \mathrm{MHz}$. The beamformer is implemented with an identical time delay- and apodization profile in both transmit and receive. The time delay profile is determined based on a desired focal point distance of $40 \mathrm{~mm}$ from the physical array. The apodization profile is a 2-D Bartlett-Hann window based on a F\# of 2.1. Fig. 3b and 3c illustrates the receive and transmit apodization profiles. From the possible 4096 elements, the SASB first stage beamformer is using 3448 elements. After the first stage beamformer, the output data is decimated with a factor 4 to $30 \mathrm{MHz}$.

The second stage beamformer is implemented using Beamformation Toolbox III, and applies a Bartlett-Hann apodization profile based on a receive F\# of 2.7 .

Parallel beamforming increases the frame rate by beamforming multiple receive line per emission. For 3-D imaging, Parallel beamforming beamforms $N \times M$ receive lines around the transmit line. For this study $4 \times 4$ lines are beamformed per emission, leading to a frame rate increase of 16 . As determined in the previous section, the maximum $f_{p r f}$ allows for 19 emissions per steering angle, leading to $19 \times 4=76$ scan lines to be beamformed per dimension.

The transmit beam should therefore be four times as wide as the receive beams. Ideally, the transmit beam should have a main-lobe width of $\alpha=60^{\circ} / 19=3.16^{\circ}$. If the focus point is placed directly under the center of the 


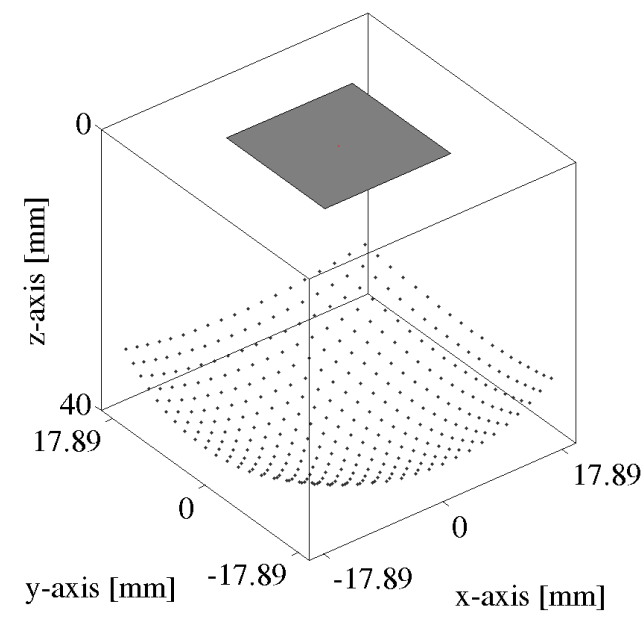

(a) Physical- and synthesized aperture

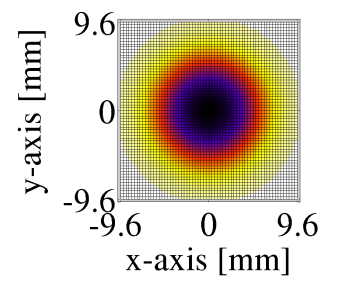

(b) SASB rx apodization

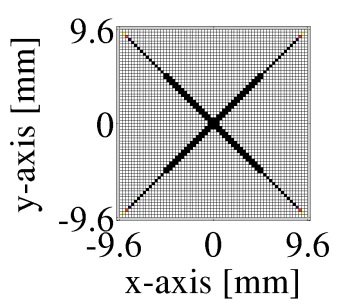

(d) PB rx apodization

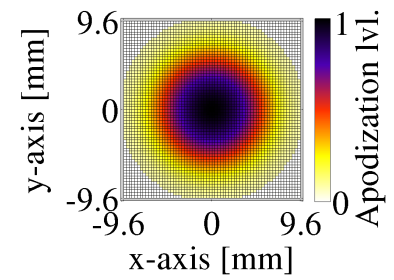

(c) SASB tx apodization

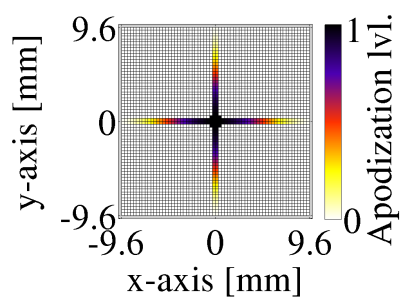

(e) PB tx apodization

Figure 3: The synthesized aperture. In (a) the physical aperture is shown with a gray square. The black dots illustrate each of the 361 focus points, that become virtual elements, in the synthesized aperture. The virtual sources are located in front of the aperture and the sound is emitted downwards, in positive z-direction. In (b) and (c) the receive- and transmit apodizations used with SASB are shown. In (d) and (e) the receive- and transmit apodizations used with Parallel Beamforing (PB) are shown.

transducer, a right-angled triangle can be drawn from the three points: the center of the active aperture, the focal point and the half-width-at-half-maximum (HWHM) of the transmit beam at the focal depth. Using this triangle and that $\mathrm{HWHM}=\mathrm{FWHM} / 2 \approx F \# \lambda / 2$, the following approximate relation can be written

$$
\tan \frac{\alpha}{2}=\frac{\mathrm{HWHM}}{r}=\frac{F \# \lambda}{2 r}=\frac{\lambda}{2 w} \quad \Leftrightarrow \quad w=\frac{\lambda}{2 \tan \frac{\alpha}{2}},
$$

where $\lambda$ is the wavelength of the emitted wave, $\alpha$ is the transmit beam width in radians, $r$ the focal depth, $w$ the aperture width, and $F \#=r / w$ is the $F$-number. In (9) tan $\frac{\alpha}{2}$ is assumed nonzero, implying that the main-lobe width is always nonzero. Inserting the values for $\lambda$ and $\alpha$ into (9) gives an aperture width of $w=9.31 \mathrm{~mm}$. With a pitch of $300 \mathrm{\mu m}$, this corresponds approximately to a 31 element wide aperture.

A circular aperture with a diameter of 31 elements contains approximately 755 elements, but the probe cable is restricted to 256 channels. It has been shown ${ }^{3,16}$ that when the number of active channels is restricted and a wide aperture is needed, the simple sparse array "Mills cross" results in one of the best ratios between imaging quality and the number of active elements used.

A 34 element wide and 4 element high cross results in exactly 256 elements and is the chosen transmit aperture. The effective size of the aperture is decreased by using a Tukey apodization. The $\psi$ parameter of the Tukey apodization which leads to the correct width, is determined by numerical optimization. The goal of the optimization routine is for the sum of the Tukey apodization coefficients to be equal to the sum of the rectangular apodization coefficients. The expression optimized is thereby

$$
\underset{\psi}{\arg \min } f(\psi)=\left(\sum_{n=1}^{N} \operatorname{Tukey}(n ; \psi)-\sum_{m=1}^{M} \operatorname{rect}(m)\right)^{2} .
$$

The 34 element wide transmit aperture is shown in Fig. 3e.

In receive, 76 lines are beamformed per dimension, giving a main lobe width of $\alpha_{R X}=60^{\circ} /(4 \times 19)=0.79^{\circ}$. Inserting $\alpha_{R X}$ in $((9))$ gives an aperture width of $37.3 \mathrm{~mm}$ or approximately 124 elements. Since the simulated 
ultrasound probe is 64 elements wide in each dimension, this receive aperture can not be implemented. Instead, the widest possible cross is implemented on the diagonals of the transducer. A Tukey apodized $64 \times \sqrt{2}=90.5$ element wide Mills Cross with $\psi=0.15$ is used as the receive aperture. The receive aperture is seen in Fig. $3 \mathrm{~d}$.

To maintain an approximately constant angular transmit beam width, the focal point is placed in the far-field. The focus distance is set to $7 \mathrm{~cm}$.

\section{SIMULATIONS}

To determine the acoustical performance over the field of view, the received field is calculated by moving a scatterer on the surface of a hemisphere with $90 \mathrm{~mm}$ radius. The scatterer is moved in azimuth, $\theta$, and elevation, $\phi$, in steps of $1^{\circ}$ from $0^{\circ}$ to $25^{\circ}$.

\subsection{C-scan imaging}

From the set of 3-D point spread functions, examples of the C-scan for the two methods are shown in Fig. 4. The figure shows that the uniformity of the C-scan created with SASB is higher than for the C-scan created with Parallel beamforming. The point spread function furthermore shows that there is little angle dependency on the visual impression of the C-scans.

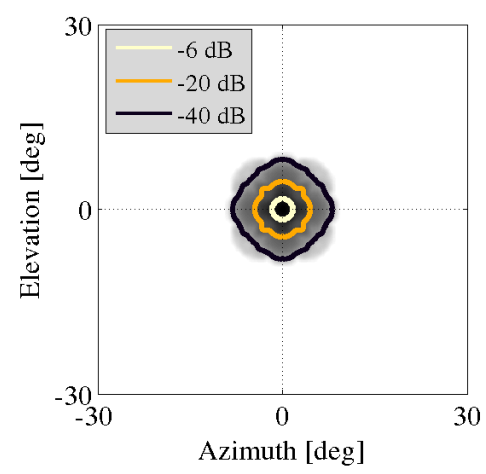

(a) SASB, $\theta=0^{\circ}, \phi=0^{\circ}$

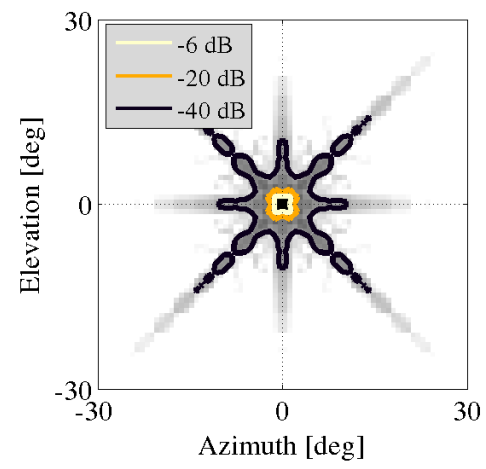

(d) PB, $\theta=0^{\circ}, \phi=0^{\circ}$

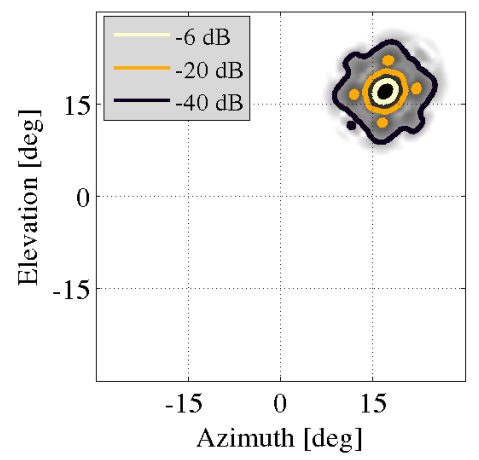

(b) SASB, $\theta=15^{\circ}, \phi=15^{\circ}$

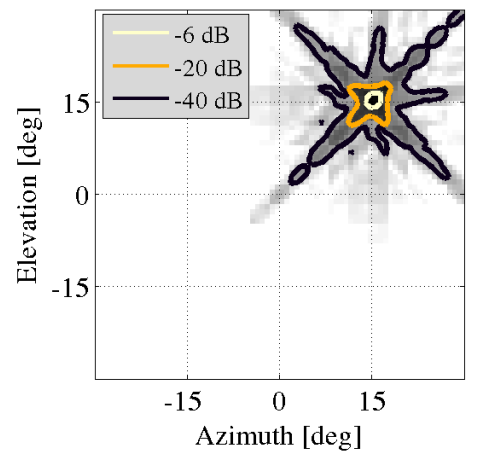

(e) PB, $\theta=15^{\circ}, \phi=15^{\circ}$

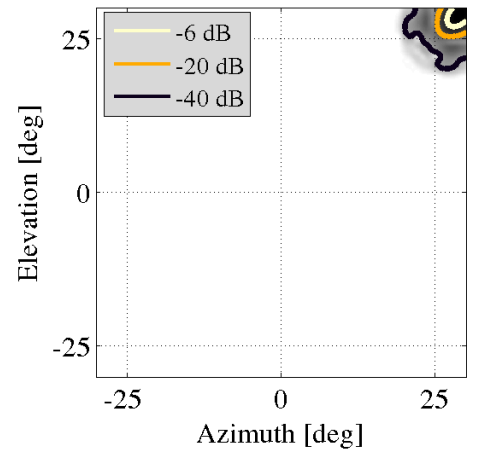

(c) SASB, $\theta=25^{\circ}, \phi=25^{\circ}$

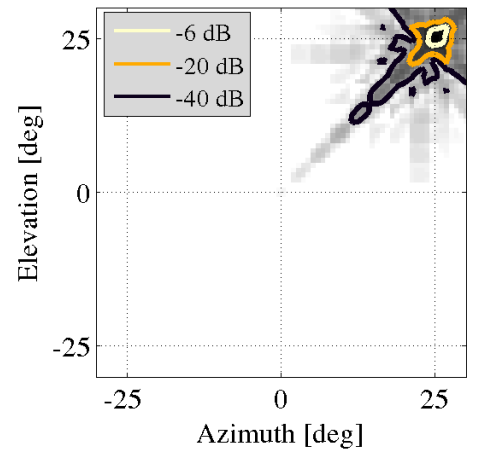

(f) PB, $\theta=25^{\circ}, \phi=25^{\circ}$

Figure 4: C-scan. (a-c) illustrate the C-scan for SASB and (d-f) Parallel beamforming. The C-scans are obtained by moving a scatterer on a hemisphere for different azimuth, $\theta$, and elevation, $\phi$, angles. The images are shown with $50 \mathrm{~dB}$ dynamic range and contour lines at $-6,-20$ and $-40 \mathrm{~dB}$.

To quantify the performance of the two methods, the detail resolution and its homogeneity is determined. The detail resolution is calculated as the full-width-half-max, FWHM, and the $-20 \mathrm{~dB}$ width, FWTM, for 64 
directions in the C-scan. The homogeneity is calculated as the coefficient of variation of the FWHM and FWTM. Fig. 5 illustrates the maximum detail resolution and the homogeneity. The graphs show that SASB has a slightly worse detail resolution, but is more homogeneous with angle.

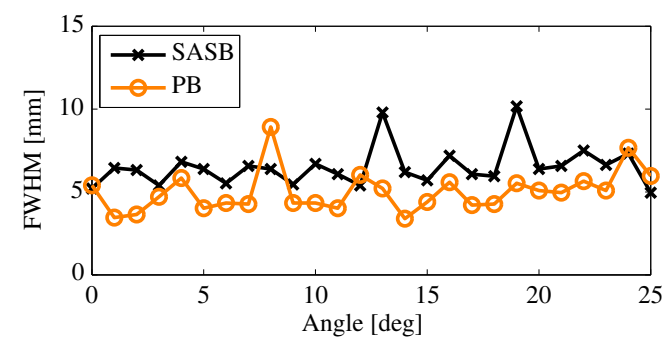

(a) FWHM

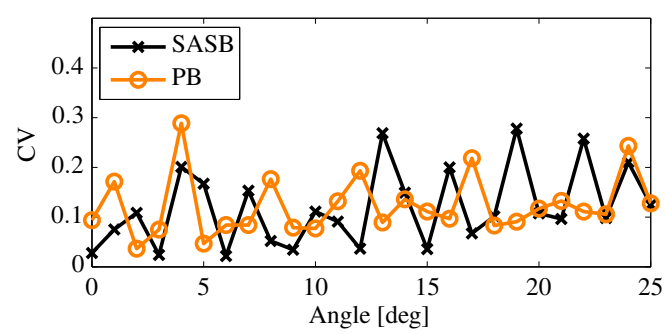

(c) FWHM coefficient of variation

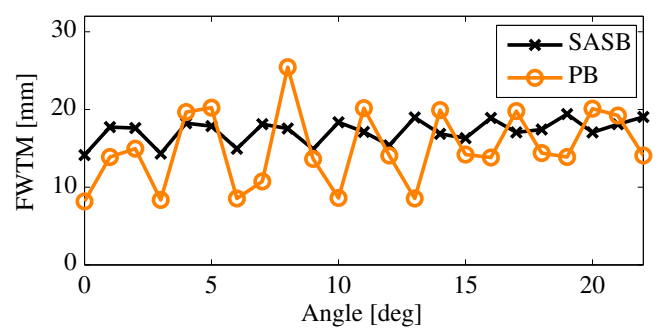

(b) FWTM

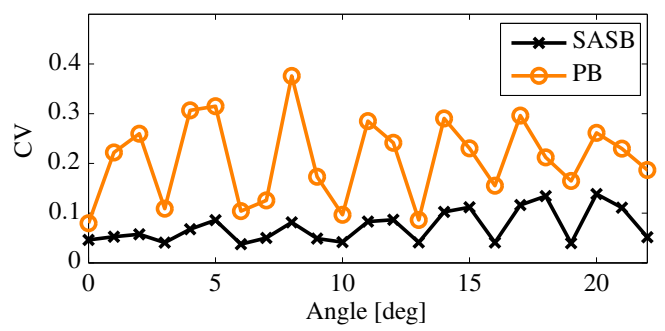

(d) FWTM, coefficient of variation

Figure 5: Performance metrics. The performance metrics are determined from the c-scan of a single scatter on a hemisphere with a radius of $90 \mathrm{~mm}$ for identical azimuth, $\theta$, and elevation, $\phi$, angles. (a-b) illustrates the detail resolution in terms of the FWHM and the FWTM. (c-d) illustrates the coefficient of variation, CV, for the FWHM and the FWTM.

\subsection{Cystic Resolution}

The Cystic Resolution describes the ability to detect an anechoic cyst in a uniform scattering medium. Specifically, the Cystic Resolution gives the intensity at the center of an anechoic cyst of a given size relative to its surrounding uniformly back-scattering medium. ${ }^{17}$ The lower the intensity at the center of the cyst, i.e., the darker it appears in the image for a given cyst size, the better imaging performance of the system. The relative intensity (RI) of the anechoic cyst was shown by Ranganathan and Walker ${ }^{18}$ to be quantized as the clutter energy to total energy ratio

$$
\mathrm{RI}(R)=\sqrt{\frac{E_{\text {out }}(R)}{E_{\text {tot }}}}=\sqrt{1-\frac{E_{\text {in }}(R)}{E_{\text {tot }}}},
$$

where $E_{i n}$ is the signal energy inside a circular region with radius, $R$, centred on the peak of the point spread function. $E_{t o t}$ is the total point spread function energy and $E_{\text {out }}$ is the point spread function energy outside the circular region. To achieve a single number from the $\mathrm{RI}(R)$-curve, the required radius to achieve a $20 \mathrm{~dB}$ relative intensity between the center of the cyst and its surroundings is determined. This is written as $R_{20 \mathrm{~dB}}$ Fig. 6 illustrates $R_{20 \mathrm{~dB}}$ as a function of identical azimuth, $\theta$, and elevation, $\phi$, angles.

To determine the $20 \mathrm{~dB}$ Cystic resolution for the 3-D point spread functions, the absolute value of the C-scans over a radial distance of $\pm 5 \mathrm{~mm}$ is summed. The absolute values of the samples along the axial dimension are summed to include out of plane energy from the 3-D point spread function. The graph shows a clear trend and a reduction in the required cyst radius to obtain a $-20 \mathrm{~dB}$ cystic resolution using SASB. The improvement is consistent with angle, and shows a reduction in cyst radius by $50 \%$. 


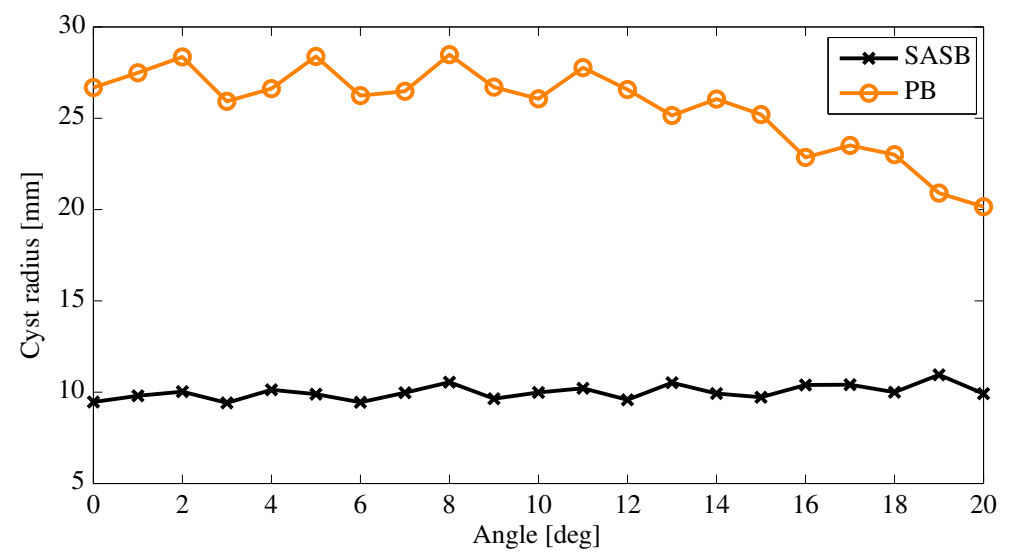

Figure 6: Cystic resolution, $R_{20 \mathrm{~dB}}$. The angle dependency of Cystic resolution is determined by moving a single scatterer on a hemisphere, with a radius of $90 \mathrm{~mm}$, for identical azimuth and elevation angles. For each angle, the summed C-scans over a radial distance of $\pm 5 \mathrm{~mm}$ is calculated and $R_{20 \mathrm{~dB}}$ is determined.

\subsection{Cyst phantom}

The simulations of an anechoic cyst are performed to link the image quality metrics to a visual comparison of a xz-plane B-mode image. A tissue mimicking phantom is created with a scatteren density of 3 scatterers $\mathrm{pr}^{\mathrm{mm}} \mathrm{m}^{3}$, to have a fully developed speckle pattern. The volume of the scatterers is positioned within $\pm 30 \mathrm{~mm}$ in the $\mathrm{x}$ and y dimensions and from $70 \mathrm{~mm}$ to $110 \mathrm{~mm}$ in the z dimension. Scatterers were removed to create a $15 \mathrm{~mm}$ spherical anechoic cyst, centered at $(\mathrm{x} ; \mathrm{y} ; \mathrm{z})=(0 ; 0 ; 90) \mathrm{mm}$. Fig. 7 shows the xz-plane B-mode image of the tissue phantom for a visual comparison of SASB and Parallel Beamforming.

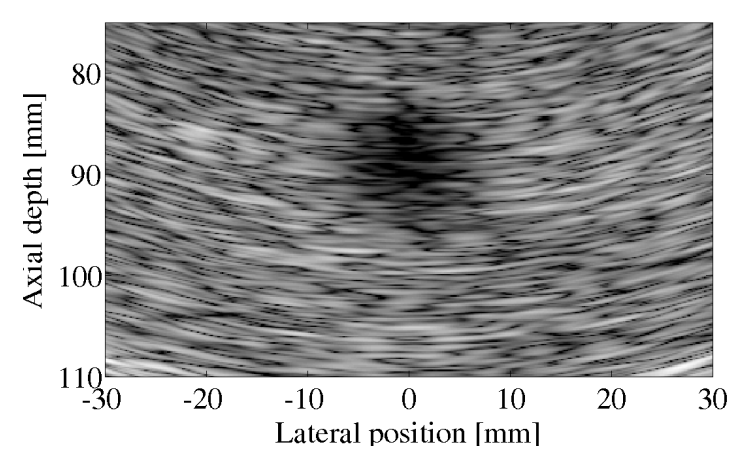

(a) SASB

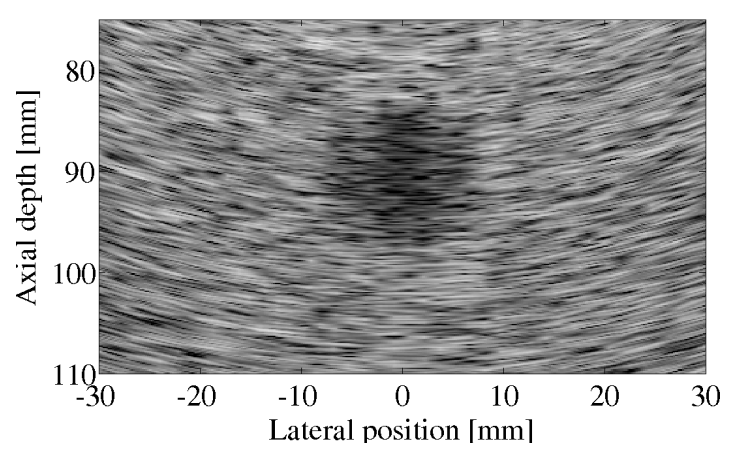

(b) Parallel Beamforming

Figure 7: xz-plane B-mode images. The two B-mode images are created with (a) SASB and (b) Parallel beamforming. They display a $15 \mathrm{~mm}$ anechoic cyst at a depth of $90 \mathrm{~mm}$, surrounded by fully developed speckle. Both images are displayed with a $50 \mathrm{~dB}$ dynamic range.

\section{CONCLUSION AND PERSPECTIVES}

This paper presented the first 3-D imaging method for a real-time ultrasound imaging system with only a single channel between the transducer and main imaging system. The huge channel reduction is achieved using a combination of synthetic aperture and sequential beamforming. The method has been compared to Parallel beamforming and the results show that the acoustic performance of SASB is less angle dependent. The Cystic resolution is shown to be more than $50 \%$ reduced with a detail resolution on the same order as Parallel 
Beamforming. It has been shown that 3-D images could be obtained for a scan depth of $10 \mathrm{~cm}$ with a view field of $60 \times 60$ degrees and a frame rate of $20 \mathrm{~Hz}$. In conclusion, SASB is an effective beamforming technique to reduce the channel count while maintaining an adequate image quality for real-time 3 -D imaging.

\section{REFERENCES}

[1] B. Delannoy, R. Torguet, C. Bruneel, E. Bridoux, J. M. Rouvaen, and H. Lasota, "Acoustical image reconstructions in parallel-processing analog electronic systems," J. Appl. Phys. 50, pp. 3153-3159, 1979.

[2] M. O'Donnell, "Efficient parallel receive beam forming for phased array imaging using phase rotation," Proc. IEEE Ultrason. Symp. , pp. 1495-1498, 1990.

[3] S. W. Smith, H. G. Pavy, and O. T. von Ramm, "High speed ultrasound volumetric imaging system - Part I: Transducer design and beam steering," IEEE Trans. Ultrason., Ferroelec., Freq. Contr. 38, pp. 100-108, 1991.

[4] O. T. von Ramm, S. W. Smith, and H. G. Pavy, "High speed ultrasound volumetric imaging system - Part II: Parallel processing and image display," IEEE Trans. Ultrason., Ferroelec., Freq. Contr. 38, pp. 109-115, 1991.

[5] C. B. Burckhardt, P.-A. Grandchamp, and H. Hoffmann, "An experimental $2 \mathrm{MHz}$ synthetic aperture sonar system intended for medical use," IEEE Trans. Son. Ultrason. 21, pp. 1-6, January 1974.

[6] J. J. Flaherty, K. R. Erikson, and V. M. Lund, "Synthetic aperture ultrasound imaging systems." United States Patent, US 3,548,642, 1967. United States Patent, US 3,548,642, 1967, Published 22 Dec 1970.

[7] M. Karaman and M. O'Donnell, "Subaperture processing for ultrasonic imaging," IEEE Trans. Ultrason., Ferroelec., Freq. Contr. 45, pp. 126-135, 1998.

[8] S. I. Nikolov, R. Dufait, A. Schoisswohl, and J. A. Jensen, "Three-dimensional real-time synthetic aperture imaging using a rotating phased array transducer," in Proc. IEEE Ultrason. Symp., pp. 1545-1548, 2002.

[9] M. Karaman, P. C. Li, and M. O'Donnell, "Synthetic aperture imaging for small scale systems," IEEE Trans. Ultrason., Ferroelec., Freq. Contr. 42, pp. 429-442, 1995.

[10] G. R. Lockwood, J. R. Talman, and S. S. Brunke, "Real-time 3-D ultrasound imaging using sparse synthetic aperture beamforming," IEEE Trans. Ultrason., Ferroelec., Freq. Contr. 45, pp. 980-988, 1998.

[11] J. Kortbek, J. A. Jensen, and K. L. Gammelmark, "Sequential beamforming for synthetic aperture imaging," Ultrasonics 53(1), pp. 1-16, 2013.

[12] M. Hemmsen, P. M. Hansen, T. Lange, J. M. Hansen, K. L. Hansen, M. B. Nielsen, and J. A. Jensen, "In vivo evaluation of synthetic aperture sequential beamforming," Ultrasound Med. Biol. 38(4), pp. 708-716, 2012.

[13] J. A. Jensen and N. B. Svendsen, "Calculation of Pressure Fields from Arbitrarily Shaped, Apodized, and Excited Ultrasound Transducers," IEEE Trans. Ultrason., Ferroelec., Freq. Contr. 39, pp. 262-267, 1992.

[14] J. A. Jensen, "Field: A program for simulating ultrasound systems," Med. Biol. Eng. Comp. 10th NordicBaltic Conference on Biomedical Imaging, Vol. 4, Supplement 1, Part 1, pp. 351-353, 1996.

[15] J. M. Hansen, M. C. Hemmsen, and J. A. Jensen, "An object-oriented multi-threaded software beamformation toolbox," in Proc. SPIE Med. Imag., 7968, pp. 79680Y 1-9, March 2011.

[16] M. Karaman, I. O. Wygant, O. Oralkan, and B. T. Khuri-Yakub, "Minimally redundant 2-D array designs for 3-D medical ultrasound imaging," IEEE Trans. Med. Imag. 7, pp. 1051-1061, jul 2009.

[17] D. Vilkomerson, J. Greenleaf, and V. Dutt, "Towards a Resolution Metric for Medical Ultrasound Imaging," in Proc. IEEE Ultrason. Symp., pp. 1405-1410, 1995.

[18] K. Ranganathan and W. F. Walker, "Cystic Resolution: A Performance Metric for Ultrasound Imaging Systems," IEEE Trans. Ultrason., Ferroelec., Freq. Contr. 54(4), pp. 782-792, 2007. 